Portland State University

PDXScholar

\title{
Elucidating Mild Cognitive Impairment: a Review of Diagnostic Research
}

Jason K. Koch

Portland State University

Follow this and additional works at: https://pdxscholar.library.pdx.edu/honorstheses

\section{Let us know how access to this document benefits you.}

\section{Recommended Citation}

Koch, Jason K., "Elucidating Mild Cognitive Impairment: a Review of Diagnostic Research" (2017).

University Honors Theses. Paper 434.

https://doi.org/10.15760/honors.431

This Thesis is brought to you for free and open access. It has been accepted for inclusion in University Honors Theses by an authorized administrator of PDXScholar. Please contact us if we can make this document more accessible: pdxscholar@pdx.edu. 
Elucidating Mild Cognitive Impairment: A review of diagnostic research

By

\section{Jason Koch}

An undergraduate honors thesis submitted in partial fulfillment of the requirements for the degree of

Bachelor of Science

in

University Honors

and

Psychology

Thesis Adviser

Dennis Swiercinsky PH.D

Portland State University 


\begin{abstract}
Mild cognitive impairment (MCI) is characterized by a level of cognitive impairment that is lower than normal for a person's age, but higher functioning than a demented person. In the majority of cases people with mild cognitive impairment revert back to normal cognitive functioning. However MCI often evolves into other more serious etiologies such as but not limited to: Alzheimer's, frontotemporal dementia, and dementia with Lewy bodies. Much effort has been made in determining the prognosis of a person with mild cognitive impairment. While treatment options are still in their exploratory stages (much of the work in this area has been fruitless) early intervention is considered to be imperative. Evidence does suggest that engaging in cognitively demanding activities (such as reading a book or playing a game of chess) can have a protective effect against the symptoms of dementia. Furthermore there are dietary changes that are correlated with better outcomes in many of the possible etiologies associated with mild cognitive impairment. This effort has led to the creation of sub-type classifications of mild cognitive impairment, some of which have been associated with an increased risk for a particular etiology. Here in, diagnostic techniques and methods are reviewed including: neuropsychological assessment, biomarkers in blood plasma, functional imaging (FDG PET \& fMRI), Volumetric and morphological imaging (using MRI), dynamical analysis of EEG and MEG data, and spontaneous speech analysis. Also explored is the link between mild cognitive impairment and depression, as well as the evolution of the construct of MCI. Through investigation of these techniques and areas of interest it becomes clear that mild cognitive impairment is a complex clinical construct arising from a variety of causes and circumstances.
\end{abstract}


Conceptualizing the neuropsychiatric diagnosis "Mild Cognitive Impairment" (MCI) has in the past twenty or so years become an increasingly important one to understand. $\mathrm{MCI}$ is a somewhat enigmatic condition in that it is difficult to detect due to its variable and typically mild—and sometimes reversible - presentation. It is critical to understand and detect because MCI may herald a variety of more serious neuropsychiatric diseases. A lack of consensus among researchers, theorists, and clinicians as to how to conceptualize, assess, and diagnose MCI accounts, somewhat, for its puzzling status. A strong conceptualization is elusive because of the myriad possible causes: sometimes transient minor cognitive fluctuations and at other times precursor of major cognitive disorder. Most people experience some cognitive decline in memory associated with aging so MCI must be clinically distinguished from normal changes. What is considered "normal" is determined psychometrically by average performance, among considerable variability, on a specific cognitive task. Relying only on comparison to average scores on such variables for differential diagnosis runs the risk of failing to accurately identify an individual case of clinically critical MCI that may predict development of a serious disorder (i.e. differentiating a false negative from a true positive diagnosis). The situation is further complicated because within a population being studied education and lifestyle variations exist, further confounding interpretation of performance scores (e.g., failing to reveal "silent" decline due to cognitive reserve occasioned by greater adaptability associated with exceptional educational background). Despite these complications, a quasi-operational definition is emerging, based on subjective symptoms of MCI. (See the current Diagnostic and Statistical Manual of Mental Disorders, APA, 2013). Still a comprehensive conceptual definition of MCI considering broad etiologies, prevalence, sub-types, disease progression, and psychometric methods awaits.

Kral (1962) was the first to approach age-related declining memory as "senescent forgetfulness." This can be considered the first attempt to characterize a clinical diagnosis approaching what is now called MCI. Building on his work, the National Institute of Mental Health introduced the term "age-related memory impairment" (AAMI) by in 1986. This designation improved on the label 
"senescent forgetfulness" by specifying that the decline in memory was abnormal for the age of the individual. However this clinical designation was largely established by comparing memory function in older adults to that of younger ones (Peterson, 2008). AAMI, focusing solely on memory, does not distinguish other domains of cognitive decline and so the International Psychogeriatric Association proposed "age related cognitive decline". In producing this expanded designation, multiple areas of cognition thought to be associated with decline in age were included. Normative values taking into account age and education level were also incorporated (Levy, 1994). Other designations and variations were proposed before the common term, 'mild cognitive impairment' was accepted as the term of choice moving forward.

The first major study indicating a level of cognitive impairment that was below normal, but not reaching decline associated with dementia came at the end of the $20^{\text {th }}$ century. Using neuropsychological evaluation, Peterson (1999) examined the performance of a cohort split into four distinct groups: demented, mildly demented, healthy controls, and those classified as having MCI. Those in the MCI group were shown to have significantly lower scores than the healthy controls, but scored consistently higher than those considered demented. Important clinically, this distinction identified people with scores between the healthy group and the mildly demented group. The level of impairment in the demented groups was designated using a "clinical dementia rating scale" score: a scale from 0-5 with 5 being the most severe score, and those not demented registering as a 0 on the scale. Anyone with a score of 1 and above was placed in the demented group; the mildly demented group scored between a .5 and a $<1$. The MCI group was not quite <.5, > 0 (Peterson, 1999).

In 2003 an international conference for MCI was convened in Stockholm in order to develop criteria for including other forms of cognitive impairment. This lead to the development of clinical subtypes: amnesiac MCI, and non-amnesiac MCI (Winblad, 2004). Building on this criteria, Peterson (2014) developed further categorizations for these sub-types relating to the number of cognitive modalities they effect: amnesiac multiple-domain MCI (affects memory and other areas of cognitive 
function), amnesiac single-domain (affects only memory), non-amnesiac multiple-domain (affects multiple domains of cognitive functioning but not memory), and non-amnesiac single-domain (only affects one domain of cognitive functioning aside from memory).

Mild cognitive impairment has recently been the focus of clinical research, due to its potential to develop into more serious conditions. Much of the motivation behind creating the diagnostic subtypes is to help clinicians and researchers sort out what variations of cognitive impairment herald which etiology. The most prevalent etiology and the focus of most research is Alzheimer's dementia (AD). $\mathrm{AD}$ is the most common etiology of each sub-type with the exception of non-amnesiac MCI with multiple domains (Busse, 2006). AD is more commonly an outcome for those MCI patients who suffer memory impairment. Progression to AD in the amnesiac MCI subcategory is roughly $10-15 \%$ (Peterson, 2008). This, as well as other diagnostic information, was the inspiration for a risk based assessment algorithm developed by Peterson (2014) and featured as Figure 1.



Figure 1.Visualization of Peterson's (2014) model of risk based assessment of patient etiology. Multiple 
diagnostic methods are employed to determine a patient's likelihood for progressing from MCI to AD.

Despite being the most common etiology and the focus of critical clinical research, Alzheimer's is far from the only condition to arise from MCI. The situation is further complicated when considering that most cases of MCI end up reverting to normal cognitive function (Paynda, 2016). Very often the condition of $\mathrm{MCI}$ is temporary, perhaps caused by something treatable like depression or chronic sleep deprivation. Nonetheless, it is important to distinguish those cases that will develop into a more serious condition. Preemptive treatment options, though currently in developing stages, are evolving fast and the need to recognize the condition of MCI before it evolves into something more serious is becoming a more pressing issue as an aging population becomes more at risk of developing dementia related conditions.

In this treatise, Mild Cognitive Impairment is explored in terms of diagnostic approaches emanating from neuropsychological assessment, functional and connectomic imaging, bio-markers, morphological study, spontaneous speech analysis, and the dynamical systems analysis of EEG/MEG data. The conceptualization and examination of MCI through the multiple lenses of diagnostic methods reveals an implacable and dynamic condition that may be the most powerful early diagnostic challenge for determining the direction of early interventions for potentially serious neurodegenerative conditions. Mild cognitive impairment is a clinical condition that arises from a variety of physiological, psychological, and life style conditions. The condition is sometimes an early warning signal of a serious condition and at other times the result of a reversible passing circumstance. As MCI becomes understood in greater depth, it will continue to be broken down into distinct variations as we further understand how to recognize the early presentation of etiological conditions. This process has already begun in the form of sub-type classification. This will here-to-for, as in many areas of scientific inquiry, be an interdisciplinary effort. This paper establishes historical perspective, explains current state-of-art clinical and technological tools, and looks into future directions for gaining greater diagnostic clarity 
and treatment interventions.

\section{Neuropsychological assessment}

Until recently the most common neuropsychological assessment tools used to detect MCI were those developed to detect AD. These tools were designed to detect much more dramatic fluctuations in cognitive ability than are present in MCI. Neuropsychological tests are those designed to assess the condition of the brain through noninvasive tasks meant to engage particular modalities of cognition. While more sophisticated cognitive assessments are sensitive enough to detect MCI, these tools are too time consuming to be performed routinely in a clinic for large numbers of patients. Until very recently the Mini Mental State Examination (MMSE) was the closest thing clinicians had to a quick and easy way to test for MCI. The MMSE is brief and samples multiple domains of cognitive ability such as episodic memory, personal orientation, and visuospatial ability. The patient is given a number of tasks, such as being asked to count back from 100 by $7 \mathrm{~s}$ or what is the date and then a score based on their performance of these tasks (Nassredine, 2005). There were other competing assessment tools that performed somewhat better than the MMSE in detecting, MCI, such as the Short Test of Mental Status (STMS). The STMS was more sensitive (in detection of MCI) than the MMSE when both were tested against one another in controlled trials (Taing-Wai, 2003). (Sensitivity refers to a test's ability to detect a particular condition.)

More recently better tools have been developed. The Montreal Cognitive Assessment (MoCA), for instance, has been shown to be significantly more sensitive and comparable in specificity (ability to distinguish between conditions) to the MMSE. In a comparison study: the MMSE had 18\% sensitivity and $100 \%$ specificity while the MoCA had $100 \%$ sensitivity and $87 \%$ specificity when detecting MCI in patients already determined to have the condition. It has been suggested that assessments focusing on semantic ability, a factor taken into account when producing the MoCA, have a greater potential for 
detecting MCI (Nassredine, 2005).

As mentioned above, the focus of clinical interest has primarily been on assessing MCI as a precursor to Alzheimer's dementia. However, effort has been made to adapt neuropsychological evaluations to also distinguish among other possible etiologies. This undertaking has largely been in the form of distinguishing sub-types. In some cases this involves focusing attention on one domain of evaluation. Patients with amnesiac MCI are considered more likely to convert to Alzheimer's disease if they present with more severe memory impairment (Peterson, 2008). When compared to controls, subjects with single domain non-amnesiac MCI mainly showed deficits in executive function when assessed using the Trail B test (Diniz, 2008). Subjects with MCI as well as vascular disease were shown to perform worse than subjects with only MCI without vascular disease in domains of speed, attention, and executive function (Norldund, 2007). Exploration of this kind contributes to developing tests that are better able to distinguish the nature of a case of MCI and to predict the future outcome of a patient. This allows for preemptive treatment for that particular condition.

There have been some attempts to build on this knowledge by augmenting existing evaluations to be more sensitive. For instance, in the previously mentioned study comparing the MMSE and STMS; the STMS was augmented with a more demanding recall component in order to make it more sensitive to MCI (Taing-Wai, 2003). Ravaglia (2005) demonstrated that, in assessing patients suspected to have MCI with multiple domains, adding a clock drawing test(CDT) to the MMSE increased the sensitivity from 50\%-40\% (either test's sensitivity respectively) to $75 \%$. This augmentation did not increase the sensitivity in other domains. In assessing likelihood of conversion one study dispensed with cognitive testing entirely. Combining a clinical dementia rating (CDR) and a questionnaire pertaining to judgment and activities asked of an informant, researchers predicted conversion correctly in $88.6 \%$ of cases examined (Daly et al, 2000). Despite this progress, neuropsychological evaluations such as the MMSE are reportedly lacking in their consistent ability to distinguish between MCI sub-types (Diniz, 2008). More research is needed to develop evaluations 
sensitive enough to distinguish between sub-types.

\section{Depression in Mild Cognitive Impairment}

The connection between depression and mild cognitive impairment has been an interesting and varied investigation. Much of the research has focused on the prevalence of depression among those who exhibited MCI. Panza (2010) found that prevalence of depression in those with MCI was about $44 \%$ in hospitalized cases (hospitalized for various reasons) and $15.7 \%$ of those in the normal population. This disparity could be attributed to selection criteria hospitalized patients being more likely to be depressed. Another area of interest concerning depression in MCI is whether or not it increases the risk of conversion (to AD). Modrego (2004) followed a cohort of those diagnosed with amnesiac MCI for a period of 3 years. Of 114 in the cohort: 59 developed dementia, $35(85 \%)$ of that group were diagnosed as having depression before conversion. It has been suggested that perhaps depression could result in MCI, but inquiry into this question is inconclusive (Defranesco, 2008). It is known, however, that depression can result in some cognitive impairment. Elderkin (2007) showed that verbal recall and set maintenance scores from neuropsychological evolution could when compared, distinguish between healthy controls and those with minor \& major depression, as well as distinguishing between those groups. Furthermore, executive function measures could separate those with minor depression from healthy controls (Elderkin, 2007). If depression were a cause of MCI this could potentially address the issue of remission. The most common outcome of MCI is, after all, reversion back to normal cognition (Petersen, 2008) and depression is normally a treatable condition.

\section{Bio-markers}

Investigation of the various underlying pathological outcomes associated with MCI reveals certain biological changes or trends that appear consistently across many etiologies. Some examples are: structural changes of specific brain areas (increase or decrease in size), reduced metabolism of specific brain areas(as seen with fMRI \& FDG PET), white matter changes in conectoemic pathways(Le Bihan, 
2001), and most notably the accumulation of miss-folded proteins into extra cellular plaques and intracellular neurofibulary tangles(Braak A. \& Braak E. 1994). Various techniques and technologies have been adapted and utilized in the investigation of these associated phenomena. Many of them, despite a lot of progress, remain unreliable and/or expensive to utilize. Baring these concerns in mind, the National Institute of Aging has issued general guidelines on what constitutes an appropriate biomarker for Alzheimer's Dementia (AD) the most common disease to arise out of MCI as well as one that shares many biological features with other etiologies associated with MCI, (Frank et al., 2003; The Ronald and Nancy Reagan Research Institute of the Alzheimer's Association and the National Institute on Aging Working Group, 1998). Ideal bio-markers for AD should: 1) detect the fundamental central nervous system pathophysiology of $\mathrm{AD}$ and be validated in confirmed neuropathological cases, 2) have a diagnostic sensitivity $>85 \%$ for detecting AD and a specificity $>75 \%$ for distinguishing between other dementias, 3) should detect any beneficial effects of disease modifying therapy, 4) be reliable, reproducible, non-invasive, simple to perform, and inexpensive, and 5) be confirmed by at least two independent studies conducted by qualified investigators with the results published in peer-reviewed journals (Franks et al., 2003). Not every bio-marker will live up to this exacting criteria, which highlights the amount of work still needed in this field.

\section{Amyloid beta/tau proteins}

Amyloid neuritic plaques and neurofibrillary tangles are the two key physical elements of Alzheimer's disease (Selkoe, 1994b). Amyloid neuritic plaques, found outside the cell, are composed primarily of two amyloid beta $(\mathrm{A} \beta$ ) proteins: $\mathrm{A} \beta$ 1-40 (containing 40 amino acids) and A $\beta 1-42$ (containing 42 amino acids (Selkoe, 1994a). Neurofibrillary tangles found inside the cell are composed of errant tauproteins that have formed hyperphosphorylated helical structures (Ihara et al, 1986). In a postmortem study involving 83 brains diagnosed as exhibiting signs of $\mathrm{AD}$, neuritic plaques and neurofibrillary tangles were found present in different areas of the brain corresponding to different intensities of 
cognitive impairment. Roughly the more impaired an individual the more dispersed the plagues and tangles. The stages can be thought of as a score ranging from 0 to VI each stage representing plagues and tangles in more areas of the brain (Braak H. \& Braak E., 1991). The early areas affected are the entorhinal cortex and hippocampal areas with later stages affecting higher cortical regions (see figure 2). MCI patients have a high variability of Braak scores. This metric is thought to be most useful when applied to cases of MCI that affect memory, such as amnesiac MCI (Stephen, 2012).
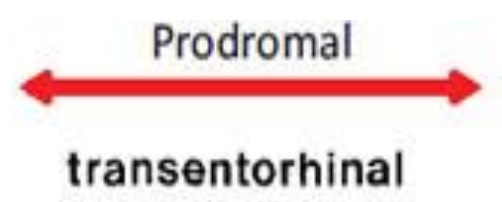
I- II

Tangles

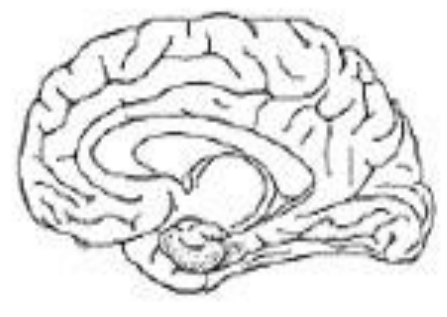

Amyloid plaques


Figure 2. Distribution of amyloid beta plaques and neurofibrulary tangles at different stages of Alzheimer's dementia (Braak E. \& Braak A., 1991)

Both $\mathrm{A} \beta$ and Tau proteins have normal healthy functions in the central nervous system and can be found ordinarily in the cerebral spinal fluid. Examining fluctuations in the presence of these proteins can potentially reveal the underlying pathology of the brain. Low levels of A $\beta 1-40$ and A $\beta$ 1-42 as well 
as high levels of Tau protein have been shown to be associated with AD ( Andreasen et al., 2001; Gloeckner et al., 2008; Sjogren et al., 2000 ). Likewise the level of A $\beta$ 1-42 has been shown to rapidly decline in cases of MCI (Andreason et al., 2001). In a meta-analysis of 17 studies examining CSF levels of $A \beta 1-42$ and 34 studies of CSF levels of Tau protein, $A \beta 1-42$ proteins were found to be significantly higher and Tau proteins were found to be significantly lower in patients with AD (Sunderland et al., 2003). Low A $\beta 1-42$ levels are also seen in other dementias such as frontotemporal dementia and dementia with Lewy bodies (Gloeckner et al., 2008), two potential etiologies of MCI. This makes CSF analysis a potential diagnostic tool for MCI, although the procedure is considered highly invasive and requires a level of expertise not widely available enough to be a realistic option.

The human body absorbs about 500ml of CSF into the blood each day (Hye et al., 2006) making blood plasma a viable, less invasive alternative for assessment of bio-makers related to neurodegenerative conditions. Unfortunately, Tau proteins are below the level of concentration to be detected in blood plasma (Tang and Kumar, 2008) but some progress has been made with A $\beta$ proteins. There are many factors that make it difficult to detect $\mathrm{A} \beta$ proteins in the blood including: age, $\mathrm{AD}$ pathology, liver catabolism, cardiovascular disease and renal excretion (urination) (Lopez et al, 2008). $\mathrm{A} \beta$ in the blood can be detected using complicated antibody assay techniques (I.E. ELISA) this however is much more difficult than in the CSF. A $\beta$ peptide levels in plasma are roughly 10 times lower than they are in the CSF and various other proteins in blood plasma can influence immunological detection (Kawarebayashi and Shoji, 2008). Despite these difficulties there has been some progress in this area of research. Locascio et al (2008) found in a longitudinal study that low levels of A $\beta$ 1-40 and 1-42 in the plasma were correlated with cognitive decline. More research is needed in this area before it can be utilized in a clinical setting, but eventually $\mathrm{A} \beta$ blood plasma levels, once they can be reliably detected, could be a reliable and non-invasive way to detect MCI.

\section{Imaging}

\section{Morphology}


Magnetic resonance imaging (MRI) utilizes strong magnetic fields and sophisticated mathematics to render a detailed three-dimensional image of the brain and other bodily organs. This enables clinicians and researchers to analyze changes in the shape, size, and volume of a rendered structure. The entorhinal cortex (ERC) and hippocampus are structures in the brain that are among the earliest affected by Alzheimer's dementia (AD) (Braak. E \& Braak. A, 1991) see figure 2. Using MRI it has been determined that a reduction in volume in these structures is associated with $\mathrm{AD}$ (Jouttonen, 1999). Naturally it was assumed that these structures could also be affected in subjects with MCI. Du. A. T. et al (2001) determined, using MRI, that there was significant loss in the volume of the ERC and hippocampus in people with MCI compared to healthy controls. The loss was not as great when compared to that of $\mathrm{AD}$ patients. It was also revealed that MCI subjects experienced an overall reduction in gray matter and an increase in ventricular volume. Vemuri (2010) in a longitudinal study comparing AD \& MCI patients with healthy controls found that increasing ventricular volume was associated with a worsening decline in cognitive and functional ability. Other studies revealed a loss of gray matter not only in the hippocampus, but also in the posterior cingulate gyrus (PCC) and even parts of the temporal neocortex (Chetelat G. 2002). Morphological and volumetric MRI assessment is emerging as a reliable diagnostic tool for MCI, but there is still work to be done for it to become a routine complement to ordinary diagnostic proceedings. While successes thus far are promising, volumetric changes are still not completely understood.

\section{Functional imaging}

Functional magnetic resonance imaging (fMRI) involves overlying the information obtained in an MRI of the brain with visual representations of the changes in the charge of hemoglobin: the protein that carries oxygen to organs in the body. When oxygen departs from the iron molecule embedded in hemoglobin it changes the protein's charge and this can be tracked by the MRI machine and overlaid on the previously rendered three-dimensional image. Simply put this allows researchers to view which parts of the brain are using more oxygen. In a typical fMRI study a reference or resting state is assessed 
and then compared to an activated state (Rombouts, 2005). The oxygen uptake is observed first in a utilized state and then in a state where it is known to be engaged in an action. This is called a blood oxygen level-dependent (BOLD) reading. An increasing area of interest for researchers is so-called "deactivations". There are certain areas in the brain with a high resting state such as: medial frontal, medial parietal and posterior cingulate gyrus (Gusnard et al. 2001). Many of these areas are associated with some of the later stages of AD (Braak E. Braak A. 1991). These areas, collectively referred to as the default mode network, appear to be deactivated when other areas of the brain are active and have been associated with perceiving environmental stimuli (Gusnard et al., 2001; Raichle et al., 2001). The default mode network has also been associate with retrieving memories and planning behaviors (Binder et al, 1999). The default mode network has been shown to be less active in those diagnosed with AD (Greicius et al, 2004). Rombouts (2005) compared 28 individuals with MCI to 18 patients with AD and 41 healthy controls. The subjects were given working and episodic memory tasks and fMRI scans. Subjects with MCI showed greater deactivation in the default mode areas than controls but not as great as AD patients. Another fMRI study comparing healthy controls to MCI and AD patients on memory as well as sensory motor tasks it was shown that individuals with MCI and AD did have decreased activation of memory related areas such as the hippocampus/medial temporal areas. However sensory motor area activation was no different from health controls (Machulda, 2003). This suggests that it is not a global decrease in activity but specific to areas related to memory.

Positron emission topography (PET) is a method of functional imaging that utilizes radioactive tracing agents that release positrons that impact with electrons and upon annihilation release two 511$\mathrm{keV} \gamma$-rays that travel in opposite directions roughly 180 degrees from each other (Newberg,2002). Most notable for the study of brains is Fluoro-2-deoxy-D-glucose positron emission tomography (FDG PET) or radiologically labeled glucose. Glucose is a sugar molecule broken down and used for energy by cells in the body. This allows researchers to use PET scanners to track which part of the brain is using more glucose than another which suggests that part of the brain is more active. 
FDG-PET scans are an area of interest as a supporting diagnostic tool for various types of dementia as well as MCI. AD patients typically show a deficit of glucose uptake (hypometabolism) in the posterior cingulate, pariototemporal cortices and frontal lobes (Masconi, 2005). Frontotemporal dementia (FTD) presents with hypometabolism in the frontal lobes early in its presentation, and then the parietal and temporal cortices in the more advanced stages of the disease (Deilh-schmid, 2007). Patients presenting with dementia with Lewy bodies (DLB) showed hypometabolism in the posterior cingulate, perietotemporal and frontal association areas, as well as the occipital cortex predominantly in the visual cortex (Minoshima, 2001). It was the presentation of hypometabolism in the visual cortex that allowed DLB to be distinguished from AD using FDG PET (Minoshima, 2001). Masconi (2008) found that $86 \%$ (101 out of 114) MCI patients scanned with FDG PET showed general cortical hypometabolism on the level indicating a neurodegenerative disease. In the same study $96 \mathrm{MCI}$ patients were found to have hypometabolism in the hippocampus alone while 92 had hypomotabolism in the hippocampus as well as the posterior cingulate cortex. Examining the FDG PET neocortical profile of a cohort of 114 MCI patients in comparison to other dementias: 29 had patterns similar to AD, 8 had patterns that reflected DLB, and 2 had patterns similar to FTD (Masconi, 2008).

\section{Diffusion tensor imaging}

One relatively new tool in the arsenal of imaging diagnostic instruments for the brain is diffusiontensor imaging (DTI). This process utilizes a powerful MRI machine to track the displacement of water molecules. Water molecules like many others are prone to random movement over time, sometimes referred to as Brownian motion. In the course of this random motion these molecules will probe their encapsulating structures at a level of resolution much smaller than what can be rendered by any available imaging device. Therefor tracking the motion and displacement of these molecules reveals detailed information about the structures that affect their movement (Le Bihan, 2001). In the case of the brain, often this imaging technique is used to assess the structural integrity of white matter fibers (Le Bihan, 2001). The fibers that connect distinct areas of the brain are often insulated with segments 
of what's called myelin. In the central nervous system these are produced by specialized glial cells called oligodendrocytes that wrap themselves around neurons as they grow. These cells maintain the integrity of neural potentials (signals) while increasing their speed by as much as 150 times. This allows for expedient communication of information between far reaching areas of the brain (Strieder, 2016).

There are several ways to analyze DTI data, the two most commonly applied metrics in the area of neurodegenerative diseases are fractional anisotropy (FA) and mean diffusivity (MD). Mean diffusivity measures the average-squared displacement of water molecules as well as the present obstacles of diffusion (Le Bihan, 2001). Fractional anisotropy determines the directionality of the fiber tracks themselves. Water will move more quickly if they are moving parallel to the structure that surrounds them. For instance water in white-matter tracks will move quickly if it is moving along the orientation of the fiber track. This allows for the determination of the orientation of the fiber track as well as revealing information about their general integrity (Le Bihan, 2001). Numerous studies have found abnormal FA and MD readings in AD patients in the corpus callosum, posterior cingulate, and the parietal \& temporal lobe (Zhang, 2007). Examination of MCI using DTI techniques has met with some success but is currently tentative as a diagnostic tool. Fellgiebel (2005) found that MCI cases showed a significant reduction in FA values in the posterior cingulum bundle as compared to healthy controls. Mean diffusivity MD values were elevated in the left side of the posterior cingulum bundle (white matter fibers projecting from the cingulate) in MCI cases compared to healthy controls. However MD \& FA were not statistically significant in comparing differences to AD (Fellgiebel, 2005). In a meta-analysis examining MCI patients Sexton (2010) found significant effect sizes (denoting differences in FA values) in the white matter of the occipital lobe, genu, frontal lobe, splenium, temporal lobe, and 4 different regions of the cingulum bundle. Many of these areas are associated with the stages of accumulation of amyloid beta plaques and neurofibrillary tangles in AD (Braak A. \& Braak E., 1991). In that same meta-analysis significant MD effect sizes were found in 6 regions in MCI 
cases: posterior cingulum, genu of the corpus callosum, temporal white matter, splenium, parietal white matter and the hippocampus (Sexton, 2011). Marked progress is being made in the formation of DTI as a diagnostic tool. One area worth exploring is correlating DTI measures with scores of neuropsychological tests. Some work has been done in this area, for instance: Fellgiebel (2005) showed that FA \& MD values of the posterior cingulum correlated significantly with delayed verbal recall test scores.

\section{Dynamical systems analysis of EEG/MEG}

An emerging trend among neuroscientists is approaching the brain as a complex nonlinear dynamical dissipative system (Stam, 2005). At its essence, this is a recognition that the brain, as a system, has emergent qualities and cannot be understood entirely by reductionist, cause-effect means. Reductionism in this context refers to examining one part of the brain at a time. Emergence is a causal property of a system that cannot be attributed to an individual element of the system (e.g. no part of a cell is alive, but in aggregate they form the smallest unit of life). For instance the magnetic waves of the brain, detectable by electroencephalograph (EEG) or magnetoencephalogram (MEG) can be thought of as emergent properties of the brain as a whole (Stam, 2005). Non-linear systems is a field concerned with (among other things) the synchronization of nonlinear complex systems. By way of EEG \& MEG it is possible to infer the likely degree of synchronicity between discrete brain regions. EEGs record different bands corresponding to different frequencies: Delta (3 Hz or below), Theta (3.5-7.5 Hz), Alpha 7.5 to $13 \mathrm{~Hz}$ ), and Beta (faster than $13 \mathrm{~Hz}$ ). Synchronization within different bands or frequencies are associated with different cognitive tasks. For instance synchronization between frontal and post central association areas are thought to be associated with working memory (Stam, 2003). Different levels of synchronicity are thought to be associated with different conditions. One would expect a brain experiencing epileptic seizures to be over synchronized while a sufferer of a neurodegenerative condition would have a lower degree of synchronization compared to normal functioning people (Stam, 2005). While readings are becoming increasingly consistent for AD, MCI 
has yet to yield results that are consistently reliable. The issue lies in distinguishing AD from MCI. Stam (2003) found that those with AD and MCI showed significantly decreased synchronization likelihood across the 14-18 and 18-22 Hz bands as compared to controls. More work is needed to develop this field, but the prospects are exciting.

\section{Speech analysis}

One area of increasing importance is the examination of spontaneous speech analysis in the diagnosis of $\mathrm{AD}$ and MCI. It has long been known that $\mathrm{AD}$ presents with discrepancies in speech such as anomia and decreased content in spontaneous speech (Cummings, 1988). Recent efforts have attempted to find ways to exploit this phenomena as a way to generate a reliable and non-invasive method of diagnosing AD (Lopez-de-Ipiña, 2015). Speech analysis research largely exists in a stage of exploration. An exact method of diagnosis or even what to look for has yet to be established. However there has been some recent success. Koing (2015) showed that using speech analysis they were able to distinguish healthy controls (HC) from MCI cases with roughly $70 \%$ accuracy, $\mathrm{HC}$ from AD with about $87 \%$ accuracy, and AD from MCI with approximately $80 \%$ accuracy. This ability to distinguish between MCI and AD is an especially exciting accomplishment. This technology has the potential to be an invaluable addition to existing efforts to render an objective diagnoses cognitive impairment.

\section{Treatment}

Much of the motivation to accurately diagnose MCI is to have an earlier indication of a more serious condition. The idea is that if a condition like AD is detected early, prospective therapies (yet to be developed) could be administered to preemptively ameliorate cognitive decline. It has been shown that cognitively stimulating activities can have a protective affect against cognitive decline and potentially reduce the risk of conversion to AD (Peterson, 2008). Reading a book or playing a game of chess are considered cognitively stimulating activities (Peterson, 2008). Additionally, decreased consumption of cholesterol, total \& saturated fats has been correlated with less cognitive decline (Peterson, 2008). An 
early indication of something like AD or other dementias would give a person time to make life style changes that could lessen the impact of a developing condition. There has been many attempts to develop a more decisive intervention, but unfortunately drug trials have seen little in the way of success (Petersen, 2007). Much of these difficulties can be attributed to our lack of understanding about the MCI construct (Peterson, 2007). As our understanding increases more possibilities for treatment will be explored.

\section{Discussion}

Mild cognitive impairment is a rapidly developing clinical diagnostic construct, attracting researchers in disciplines from psychology to applied mathematics. Still, there are gaps in our understanding of MCI. The biomarkers we know to be associated with $\mathrm{MCI}$ (such as $\mathrm{A} \beta$ plaques and neurofibrillary tangles) have been examined through a variety of different means. However it is clear especially considering the failed attempts at intervention (Peterson, 2007) that there are factors involved to which we are unaware. Some scientists are embracing this and taking on more explorative methods of research. For instance some methods in proteomics (the study of proteins), such as mass spectrometry allow for an inventory of many different proteins in blood plasma and serum (Song, 2009). This allows researchers to explore the physiology of a person with $\mathrm{AD}$ or MCI without focusing on just one specific bio-marker. Developing areas such as dynamical systems analysis of EEGs or automatic speech analysis offer exciting possibilities for the future. One can imagine a time when we know exactly what proteins to look for in blood plasma and what aberrations in speech betray an inchoate neurodegenerative condition. In such a time, an app on your phone could alert a person to go see a physician long before even minor symptoms develop. Such a time is a long way off. There is much more to learn about the diagnoses and especially the, treatment of MCI.

As the population ages, $\mathrm{MCI}$ is becoming a more important area of research. One area in need of much attention is prevalence in the population. MCI has been problematic in this area for a number of reasons. One is a lack of consensus in the criteria that make up MCI. 
MCI remains a complex construct, even more so after examining the purposed mechanisms and theories of its pathology. As is the case with any complex phenomena, unraveling its mysteries will be an interdisciplinary endeavor. MCI should not be viewed as a condition unto itself, it is not a cause of symptoms. It can be thought of, in many cases, as a transient set of symptoms arrived at for a variety of reasons and in the course of a developing prognosis. Future research on MCI should focus on understanding the dynamics of the underlying condition. It is in this understanding that we can learn all the causes of MCI, for indeed there are more than one and they are not necessarily all related. A smart approach would be to make an effort to correlate the presentation of neuropsychological symptoms with underlying physiological markers. For instance the effort to associate different cognitive tasks with desynchronization of brain regions or to correlate executive function to vascular disease is a step in the right direction. Once these connections are made, they can be grouped into clusters of symptoms. This process will eventually lead to more distinct and useful subtypes of MCI. Throughout this process it is necessary to explore convenient and affordable methods of detecting these physiological indicators. It is through this iterative process that MCI will eventually become a useful construct in determining the prognosis of its sufferers. $\mathrm{MCI}$ in its current form is more appropriately thought of as a target for efforts in research than a clinical diagnosis. Put another way MCI is not an answer, it is another question on the path to an answer. 
A Thomann, P., Seidl, U., Brinkmann, J., Hirjak, D., Traeger, T., Christian Wolf, R.,\& Schroder, J (2012). Hippocampal morphology and autobiographic memory in mild cognitive impairment and Alzheimer's disease. Current Alzheimer Research, 9(4), 507-515.

Andreasen, N., Minthon, L., Davidsson, P., Vanmechelen, E., Vanderstichele, H.,Winblad, B., Blennow, K.,( 2001). Evaluation of CSF-tau and CSF-Abeta42 as diagnostic markers for Alzheimer disease in clinical practice. Arch. Neurol. 58, 373- 379.

Binder JR, Frost JA, Hammeke TA, Bellgowan PS, Rao SM, Cox RW (1999): Conceptual processing during the conscious resting state. A functional MRI study. J Cogn Neurosci 11:80-95.

Braak, H., \& Braak, E. (1991). Neuropathological stageing of Alzheimer-related changes. Acta neuropathologica, 82(4), 239-259.

Bruscoli, M., \& Lovestone, S. (2004). Is MCI really just early dementia? A systematic review of conversion studies. International Psychogeriatrics, 16(02), 129-140.

Busse, A., Hensel, A., Gühne, U., Angermeyer, M. C., \& Riedel-Heller, S. G. (2006). Mild cognitive impairment Long-term course of four clinical subtypes. Neurology, 67(12), 2176-2185. https://doi.org/10.1212/01.wnl.0000249117.23318.e1

Chetelat G, Desgranges B, de la Sayette V, Viader F, Eustache F, Baron JC (2002): Mapping gray matter loss with voxel-based morphometry in mild cognitive impairment. Neuroreport 13: 1939-1943.

Choi, J., Malakowsky, C.A., Talent, J.M., Conrad, C.C., Gracy, R.W.,(2002). Identification of oxidized plasma proteins in Alzheimer's disease. Biochem. Biophys. Res. Commun. 293, 1566- 1570.

Cummings, J. L., Darkins, A., Mendez, M., Hill, M. A., \& Benson, D. F. (1988). Alzheimer's disease and Parkinson's disease Comparison of speech and language alterations. Neurology, 38(5), 680680.

Daly, E., Zaitchik, D., Copeland, M., Schmahmann, J., Gunther, J., et al. (2000). Predicting conversion to Alzheimer disease using standardized clinical information. Archives of Neurology, 57, 675680.

DeCarli, C., Mungas, D., Harvey, D., Reed, B., Weiner, M., Chui, H., \& Jagust, W. (2004). Memory impairment, but not cerebrovascular disease, predicts progression of MCI to dementia. Neurology, 63(2), 220-227. https://doi.org/10.1212/01.WNL.0000130531.90205.EF

Defrancesco, M., Marksteiner, J., Deisenhammer, E. A., Hinterhuber, H., \& Weiss, E. M. (2008). Association of mild cognitive impairment (MCI) and depression. Neuropsychiatrie: Klinik, Diagnostik, Therapie und Rehabilitation: Organ der Gesellschaft Osterreichischer Nervenarzte und Psychiater, 23(3), 144-150.

Diniz, B. S., Nunes, P. V., Yassuda, M. S., Pereira, F. S., Flaks, M. K., Viola, L. F., ... Forlenza, O. V. (2008). Mild cognitive impairment: cognitive screening or neuropsychological assessment? Revista Brasileira de Psiquiatria, 30(4), 316-321. https://doi.org/10.1590/S151644462008000400003 
Diehl-Schmid, J., Grimmer, T., Drzezga, A., Bornschein, S., Riemenschneider, M., Förstl, H.,\& Kurz, A. (2007). Decline of cerebral glucose metabolism in frontotemporal dementia: a longitudinal 18F-FDG-PET-study. Neurobiology of aging, 28(1), 42-50.

Douaud, G., Jbabdi, S., Behrens, T. E., Menke, R. A., Gass, A., Monsch, A. U., \& Smith, S. (2011). DTI measures in crossing-fibre areas: increased diffusion anisotropy reveals early white matter alteration in MCI and mild Alzheimer's disease. Neuroimage, 55(3), 880-890.

Du, A. T., Schuff, N., Amend, D., Laakso, M. P., Hsu, Y. Y., Jagust, W. J.,\& Chui, H. C. (2001). Magnetic resonance imaging of the entorhinal cortex and hippocampus in mild cognitive impairment and Alzheimer's disease. Journal of Neurology, Neurosurgery \& Psychiatry, 71(4), 441-447.

Elderkin-Thompson, V., Mintz, J., Haroon, E., Lavretsky, H., \& Kumar, A. (2007). Executive dysfunction and memory in older patients with major and minor depression. Archives of Clinical Neuropsychology, 22(2), 261-270.

Fellgiebel, A., Müller, M. J., Wille, P., Dellani, P. R., Scheurich, A., Schmidt, L. G., \& Stoeter, P. (2005). Color-coded diffusion-tensor-imaging of posterior cingulate fiber tracts in mild cognitive impairment. Neurobiology of aging, 26(8), 1193-1198.

Frank, R.A., Galasko, D., Hampel, H., Hardy, J., de Leon, M.J., Mehta, P.D., Rogers, J., Siemers, E., Trojanowski, J.Q., (2003). Biological markers for therapeutic trials in Alzheimer's disease. Proceedings of the biological markers working group; NIA initiative on neuroimaging in Alzheimer's disease. Neurobiol. Aging 24, 521-536.

Gloeckner, S.F., Meyne, F., Wagner, F., Heinemann, U., Krasnianski, A., Meissner, B., Zerr, I., (2008). Quantitative analysis of transthyretin, tau and amyloid-beta in patients with dementia. J. Alzheimers Dis. 14, 17- 25.

Gollin, P.A., Kalaria, R.N., Eikelenboom, P., Rozemuller, A., Perry, G., (1992). Alpha 1-antitrypsin and alpha 1-antichymotrypsin are in the lesions of Alzheimer's disease. Neuroreport 3, 201- 203.

Greicius MD, Krasnow B, Reiss AL, Menon V (2003): Functional connectivity in the resting brain: a network analysis of the default mode hypothesis. Proc Natl Acad Sci U S A 100: 253-258.

Greicius MD, Srivastava G, Reiss AL, Menon V (2004): Default-mode network activity distinguishes Alzheimer's disease from healthy aging: evidence from functional MRI. Proc Natl Acad Sci U S A 101: 4637-4642.

Gusnard DA, Akbudak E, Shulman GL, Raichle ME (2001): Medial prefrontal cortex and selfreferential mental activity: relation to a default mode of brain function. Proc Natl Acad Sci U S A 98: 4259-4264.

Henneman, W. J. P., Sluimer, J. D., Barnes, J., Van Der Flier, W. M., Sluimer, I. C., Fox, N. C., ... \& Barkhof, F. (2009). Hippocampal atrophy rates in Alzheimer disease Added value over whole brain volume measures. Neurology, 72(11), 999-1007. 
Hiltunen, M., van Groen, T., \& Jolkkonen, J. (2009). Functional roles of amyloid- $\beta$ protein precursor and amyloid- $\beta$ peptides: evidence from experimental studies. Journal of Alzheimer's Disease, 18(2), 401-412.

Hogan, D. B. and Ebly, E. M. (2000). Predicting who will develop dementia in a cohort of Canadian seniors. Canadian Journal of Neurological Science, 27, 18-24

Huang, C., Wahlund, L. O., Dierks, T., Julin, P., Winblad, B., et al. (2000). Discrimination of Alzheimer's disease and mild cognitive impairment by equivalent EEG sources: a crosssectional and longitudinal study. Clinical Neurophysiology, 111, 1961-1967.

Hye, A., Lynham, S., Thambisetty, M., Causevic, M., Campbell, J., Byers, H.L., Hooper, C., Rijsdijk, F., Tabrizi, S.J., Banner, S., Shaw, C.E., Foy, C., Poppe, M., Archer, N., Hamilton, G., Powell, J., Brown, R.G., Sham, P., Ward, M., Lovestone, S.,(2006). Proteome-based plasma biomarkers for Alzheimer's disease. Brain 129, 3042-3050.

Ihara, Y., Nuknia, N., Miura, R., \& Ogawara, M. (1986). Phosphorylated tau protein is integrated into paired helical filaments in Alzheimer's disease. The Journal of Biochemistry, 9 9(6), $1807-$ 1810.

Jack, C. R., Petersen, R. C., Xu, Y. C., O’Brien, P. C., Smith, G. E., Ivnik, R. J., .\& Kokmen, E.(1999). Prediction of AD with MRI-based hippocampal volume in mild cognitive impairment. Neurology, 52(7), 1397-1397.

Jun, G., Naj, A. C., Beecham, G. W., Wang, L. S., Buros, J., Gallins, P. J., ... \& Inzelberg, R. (2010). Meta-analysis confirms CR1, CLU, and PICALM as Alzheimer disease risk loci and reveals interactions with APOE genotypes. Archives of neurology, 67(12), 1473-1484.

Juottonen, K., Laakso, M. P., Partanen, K., \& Soininen, H. (1999). Comparative MR analysis of the entorhinal cortex and hippocampus in diagnosing Alzheimer disease. American Journal of Neuroradiology, 20(1), 139-144.

Kawarabayashi, T., Shoji, M., (2008). Plasma biomarkers of Alzheimer's disease. Curr. Opin. Psychiatry 21, 260- 267.

Kral VA. Senescent forgetfulness: benign and malignant.(1962) Can Med Assoc J. 1962;86:257-260.

König, A., Satt, A., Sorin, A., Hoory, R., Toledo-Ronen, O., Derreumaux, A., ... \& David, R. (2015). Automatic speech analysis for the assessment of patients with predementia and Alzheimer's disease. Alzheimer's \& Dementia: Diagnosis, Assessment \& Disease Monitoring, 1(1), 112-124.

Koldamova, R.P, Letterou, I.M., Lefterova, M.I., Lazo, J.S., (2001). Apolipoprotein A-I directly interacts with amyloid precursor protein and inhibits A beta aggregation and toxicity. Biochemistry 40, 3553-3560.

Le Bihan, D., Mangin, J. F., Poupon, C., Clark, C. A., Pappata, S., Molko, N., \& Chabriat, H. (2001). Diffusion tensor imaging: concepts and applications. Journal of magnetic resonance imaging, 1 3(4), 534-546. 
Leung, K. K., Barnes, J., Ridgway, G. R., Bartlett, J. W., Clarkson, M. J., Macdonald, K., ... \& Alzheimer's Disease Neuroimaging Initiative. (2010). Automated cross-sectional and longitudinal hippocampal volume measurement in mild cognitive impairment and Alzheimer's disease. Neuroimage, 51(4), 1345-1359.

Levy R. Aging-associated cognitive decline. Int Psychogeriatr. 1994;6:63-68.

Liao, P.-c., Yu, L., Kuo, C.-C., Lin, C., Kuo, Y.-M., (2007). Proteomics analysis of plasma for potential biomarkers in the diagnosis of Alzheimer's disease. Proteomics — Clinical Applications 1, 506512.

Likeman, M., Anderson, V.M., Stevens, J.M., Waldman, A.D., Godbolt, A.K., Frost, C., Rossor, M.N., Fox, N.C., (2005). Visual assessment of atrophy on magnetic resonance imaging in the diagnosis of pathologically confi rmed young-onset dementias. Arch. Neurol. 62, 1410- 1415.

Litvan, I., Aarsland, D., Adler, C. H., Goldman, J. G., Kulisevsky, J., Mollenhauer, B., Weintraub, D. (2011). MDS task force on mild cognitive impairment in Parkinson's disease: Critical review of PD-MCI. Movement Disorders, 26(10), 1814-1824. https://doi.org/10.1002/mds.23823

Locascio, J.J., Fukumoto, H., Yap, L., Bottiglieri, T., Growdon, J.H., Hyman, B.T., Irizarry, M.C., (2008). Plasma amyloid beta-protein and C-reactive protein in relation to the rate of progression of Alzheimer disease. Arch. Neurol. 5, 776- 785.

Lopez-de-Ipiña, K., Alonso, J. B., Solé-Casals, J., Barroso, N., Henriquez, P., Faundez-Zanuy, M., ... \& Eguiraun, H. (2015). On automatic diagnosis of Alzheimer's disease based on spontaneous speech analysis and emotional temperature. Cognitive Computation, 7(1), 44-55.

Lopez, O. L., Kuller, L. H., Mehta, P. D., Becker, J. T., Gach, H. M., Sweet, R. A., ... \& DeKosky, S. T. (2008). Plasma amyloid levels and the risk of AD in normal subjects in the Cardiovascular Health Study. Neurology, 70(19), 1664-1671.

Lopez, M.F., Mikulskis, A., Kuzdzal, S., Bennett, D.A., Kelly, J., Golenko, E., DiCesare, J., Denoyer, E., Patton, W.F., Ediger, R., Sapp, L., Ziegert, T., Lynch, C., Kramer, S., Whiteley, G.R., Wall, M.R., Mannion, D.P., Della Cioppa, G., Rakitan, J.S., Wolfe, G.M., (2005). High-resolution serum proteomic profiling of Alzheimer disease samples reveals disease-specific, carrierprotein-bound mass signatures. Clin. Chem. 51, 1946- 1954.

Machulda, M. M., Ward, H. A., Borowski, B., Gunter, J. L., Cha, R. H., O’brien, P. C., ... \& Ivnik, R. J. (2003). Comparison of memory fMRI response among normal, MCI, and Alzheimer's patients. Neurology, 61(4), 500-506.

Medina, D., Urresta, F., Gabrieli, J. D., Moseley, M., Fleischman, D., Bennett, D. A., ... \& Stebbins, G. T. (2006). White matter changes in mild cognitive impairment and AD: A diffusion tensor imaging study. Neurobiology of aging, 27(5), 663-672.

Minoshima, S., Foster, N. L., Sima, A. A., Frey, K. A., Albin, R. L., \& Kuhl, D. E. (2001). Alzheimer's disease versus dementia with Lewy bodies: cerebral metabolic distinction with autopsy confirmation. Annals of neurology, 50(3), 358-365.

Modrego, P. J., \& Ferrández, J. (2004). Depression in patients with mild cognitive impairment 
increases the risk of developing dementia of Alzheimer type: a prospective cohort study. Archives of neurology, 61(8), 1290-1293.

Mosconi, L., Tsui, W. H., Herholz, K., Pupi, A., Drzezga, A., Lucignani, G.,Leon, M. J. de. (2008). Multicenter Standardized 18F-FDG PET Diagnosis of Mild Cognitive Impairment, Alzheimer's Disease, and Other Dementias. Journal of Nuclear Medicine, 49(3), 390-398. https://doi.org/10.2967/jnumed.107.045385

Mosconi, L. (2005). Brain glucose metabolism in the early and specific diagnosis of Alzheimer's disease. European journal of nuclear medicine and molecular imaging, 32(4), 486-510.

Nasreddine, Z. S., Phillips, N. A., Bédirian, V., Charbonneau, S., Whitehead, V., Collin, I., ... \& Chertkow, H. (2005). The Montreal Cognitive Assessment, MoCA: a brief screening tool for mild cognitive impairment. Journal of the American Geriatrics Society, 53(4), 695-699.

Newberg, A., Alavi, A., \& Reivich, M. (2002, January). Determination of regional cerebral function with FDG-PET imaging in neuropsychiatric disorders. In Seminars in nuclear medicine (Vol. 32, No. 1, pp. 13-34). WB Saunders.

Nielsen, H.M., Minthon, L., Londos, E., Blennow, K., Miranda, E., Perez, J., Crowther, D.C., Lomas, D.A., Janciauskiene, S.M., (2007). Plasma and CSF serpins in Alzheimer disease and dementia with Lewy bodies. Neurology 69, 1569- 1579.

Nordlund, A., Rolstad, S., Klang, O., Lind, K., Hansen, S., \& Wallin, A. (2007). Cognitive profiles of mild cognitive impairment with and without vascular disease. Neuropsychology, 21(6), 706712. https://doi.org/10.1037/0894-4105.21.6.706

Panza, F., Frisardi, V., Capurso, C., D'introno, A., Colacicco, A. M., Imbimbo, B. P., ... \& Capurso, A. (2010). Late-life depression, mild cognitive impairment, and dementia: possible continuum?. The American Journal of Geriatric Psychiatry, 18(2), 98-116.

Petersen, R. C., Caracciolo, B., Brayne, C., Gauthier, S., Jelic, V., \& Fratiglioni, L. (2014). Mild cognitive impairment: a concept in evolution. Journal of internal medicine, 275(3), 214-228.

Petersen, R. C. (2007). MCI treatment trials: failure or not?. The Lancet Neurology, 6(6), 473-475.

Petersen, R. C., Smith, G. E., Waring, S. C., Ivnik, R. J., Tangalos, E. G., \& Kokmen, E. (1999). Mild cognitive impairment: clinical characterization and outcome. Archives of neurology, 56(3), 303308 .

Petersen, R. C., \& Negash, S. (2008). Mild cognitive impairment: an overview. CNS spectrums, 13(01), 45-53.

Pihlajamaki, M., Jauhiainen, A. M., \& Soininen, H. (2009). Structural and functional MRI in mild cognitive impairment. Current Alzheimer Research, 6(2), 179-185.

Poljak, A., Sachdev, P., Smythe, G.A., 2006. Causes and diagnosis of Alzheimer's disease: a proteomics approach. Current Proteomics 81- 112. 
Potempa, J., Korzus, E., Travis, J., (1994). The serpin superfamily of proteinase inhibitors: structure, function, and regulation. J. Biol. Chem. 269, 15957- 15960.

Raichle ME, MacLeod AM, Snyder AZ, Powers WJ, Gusnard DA, Shulman GL (2001): A default mode of brain function. Proc Natl Acad Sci U S A 98: 676-682

Ravaglia G, Forti P, Maioli F, Servadei L, Martelli M, Brunetti N, Bastagli L, Mariani E. (2005) Screening for mild cognitive impairment in elderly ambulatory patients with cognitive complaints. Aging Clin ～Exp Res. 2005;17(5):374-9

Rombouts, S. A., Barkhof, F., Goekoop, R., Stam, C. J., \& Scheltens, P. (2005). Altered resting state networks in mild cognitive impairment and mild Alzheimer's disease: an fMRI study. Human brain mapping, 26(4), 231-239.

Selkoe, D.J., 1994a. Normal and abnormal biology of the beta-amyloid precursor protein. Annu. Rev. Neurosci. 17, 489- 517.

Selkoe, D.J., 1994b. Alzheimer's disease: a central role for amyloid. J. Neuropathol. Exp. Neurol. 53, $438-447$.

Sexton, C. E., Kalu, U. G., Filippini, N., Mackay, C. E., \& Ebmeier, K. P. (2011). A meta-analysis of diffusion tensor imaging in mild cognitive impairment and Alzheimer's disease. Neurobiology of aging, 32(12), 2322-e5.

Sihlbom, C., Davidsson, P., Sjogren, M., Wahlund, L.O., Nilsson, C.L., 2008. Structural and quantitative comparison of cerebrospinal fluid glycoproteins in Alzheimer's disease patients and healthy individuals. Neurochem. Res. 33, $1332-1340$.

Sjogren, M., Minthon, L., Davidsson, P., Granerus, A.K., Clarberg, A., Vanderstichele, H.,Vanmechelen, E., Wallin, A., Blennow, K., (2000). CSF levels of tau, beta-amyloid(1-42) and GAP-43 in frontotemporal dementia, other types of dementia and normal aging. J. Neural. Transm. 107, 563- 579.

Smach, M.A., Charfeddine, B., Lammouchi, T., Harrabi, I., Ben Othman, L., Dridi, H., Bennamou, S., Limem, K., (2008). CSF beta-amyloid 1- 42 and tau in Tunisian patients with Alzheimer's disease: the effect of APOE epsilon4 allele. Neurosci. Lett. 440, 145- 149.

Song, F., Poljak, A., Smythe, G. A., \& Sachdev, P. (2009). Plasma biomarkers for mild cognitive impairment and Alzheimer's disease. Brain research reviews, 61(2), 69-80.

Stam, C. J. (2005). Nonlinear dynamical analysis of EEG and MEG: review of an emerging field. Clinical neurophysiology, 116(10), 2266-2301.

Stephan, B. C. M., Hunter, S., Harris, D., Llewellyn, D. J., Siervo, M., Matthews, F. E., \& Brayne, C. (2012). The neuropathological profile of mild cognitive impairment (MCI): a systematic review. Molecular psychiatry, 17(11), 1056-1076.

Striedter, G. F. (2016). Neurobiology: a functional approach. New York: Oxford University Press. 
Sunderland, T., Linker, G., Mirza, N., Putnam, K.T., Friedman, D.L., Kimmel, L.H., Bergeson, J., Manetti, G.J., Zimmermann, M., Tang, B., Bartko, J.J., Cohen, R.M., (2003). Decreased betaamyloid1-42 and increased tau levels in cerebrospinal fluid of patients with Alzheimer disease. JAMA 289, 2094- 2103.

Tang, B.L., Kumar, R., (2008). Biomarkers of mild cognitive impairment and Alzheimer's disease. Ann. Acad. Med. Singapore 37, 406-410.

Tang-Wai, D. F., Knopman, D. S., Geda, Y. E., Edland, S. D., Smith, G. E., Ivnik, R. J., Petersen, R. C. (2003). Comparison of the Short Test of Mental Status and the Mini-Mental State Examination in Mild Cognitive Impairment. Archives of Neurology, 60(12), 1777-1781. https://doi.org/10.1001/archneur.60.12.1777

Winblad, B., Palmer, K., Kivipelto, M., Jelic, V., Fratiglioni, L., Wahlund, L. O., ... \& Arai, H. (2004). Mild cognitive impairment-beyond controversies, towards a consensus: report of the International Working Group on Mild Cognitive Impairment. Journal of internal medicine, 256(3), 240-246.

Vemuri, P., Wiste, H. J., Weigand, S. D., Knopman, D. S., Trojanowski, J. Q., Shaw, L. M., ... \& Jack, C. R. (2010). Serial MRI and CSF biomarkers in normal aging, MCI, and AD. Neurology, 75(2), 143-151.

Zhang, Y., Schuff, N., Jahng, G. H., Bayne, W., Mori, S., Schad, L., ... \& Chui, H. (2007). Diffusion tensor imaging of cingulum fibers in mild cognitive impairment and Alzheimer disease. Neurology, 68(1), 13-19. 